\title{
REGULAR OPERATOR CONVERGENCE AND NONLINEAR EQUATIONS INVOLVING NUMERICAL RANGES
}

\author{
RAM U. VERMA
}

(Communicated by Dale Alspach)

\begin{abstract}
Regular operator approximation theory, based on the work of Anselone and Lei (1986), is generalized to the case of strongly accretive operators and applied to nonlinear equations involving the generalized Zarantonello numerical ranges
\end{abstract}

\section{INTRODUCTION}

Recently Anselone and Lei [4] studied the regular operator approximation theory, which heavily relies on inverse compactness principles and provides a convenient general framework for the convergence of approximation solutions; then the existence of solutions follows in a natural manner. They applied this theory to the solvability of equations involving strongly monotone operators, and, as a result, they illustrated the theory to nonlinear integral equations of Urysohn and Hammerstein types. For a detailed account on regular approximation theory, we refer to $[1-6,15]$.

In this paper our aim is to generalize the results of Anselone and Lei [4] to the case of nonlinear equations involving strongly accretive operators in a slightly different setting. The obtained results are applied to the equations involving the theory of the generalized Zarantonello numerical ranges.

Now we need to recall some definitions. Let $X$ and $Y$ be two Banach spaces. Let $N=\{1,2, \ldots\}$ with infinite subsets $N^{\prime}, N^{\prime \prime}, \ldots$ Let $\left\{x_{n}\right\}=\left\{x_{n}: n \in\right.$ $N\} \subset X$, and let $S_{n} \subset X$ for $n \in N$. Then $\left\{x_{n}\right\}^{*}=\left\{x \in X: x_{n} \rightarrow x, n \in N^{\prime}\right\}$ and $\left\{S_{n}\right\}^{*}=\left\{x \in X: x_{n} \rightarrow x, x_{n} \in S_{n}, n \in N^{\prime}\right\}$ are called the sets of all cluster points (limits of sequences).

A sequence $\left\{x_{n}\right\}$ is said to be $d$-compact (discretely compact) if every subsequence has a convergent subsequence. Alternatively, $\left\{x_{n}\right\}$ is $d$-compact if $\left\{x_{n}: n \in N^{\prime}\right\}^{*} \neq \varnothing$ for $n \in N^{\prime}$. Similarly, $\left\{S_{n}\right\}$, is called $d$-compact if every subsequence $\left\{x_{n} \in S_{n}: n \in N^{\prime}\right\}$ has a convergent subsequence. Alternatively, $\left\{S_{n}\right\}$ is $d$-compact if $\left\{S_{n}: n \in N^{\prime}\right\}^{*} \neq \varnothing$ for all $N^{\prime}$ such that $S_{n} \neq \varnothing$ for $n \in N^{\prime}$. If $S_{n}=\varnothing$ for $n \in N$, then $\left\{S_{n}\right\}$ is trivially $d$-compact.

A set sequence $\left\{S_{n}\right\}$ is said to converge to a set $S\left(S_{n} \rightarrow S\right.$ as $\left.n \rightarrow \infty\right)$ if any $\varepsilon$-neighboorhood of $S$ contains $S_{n}$ for all $n$ sufficiently large. Such a

Received by the editors April 21, 1993 and, in revised form, September 28, 1993.

1991 Mathematics Subject Classification. Primary 65J15; Secondary 47H17. 
limit is not unique, that is, $S_{n} \rightarrow S \subset S^{\prime} \Rightarrow S_{n} \rightarrow S^{\prime}$. Similarly, $S_{n}=\varnothing$ for $n \in N \Rightarrow S_{n} \rightarrow S$ for all $S \subset X, S_{n} \rightarrow \varnothing \Rightarrow S_{n}=\varnothing$ for $n$ sufficiently large, and $S_{n} \neq \varnothing$ for $n \in N^{\prime}, S_{n} \rightarrow S \Rightarrow S \neq \varnothing$. The connection between the set convergence and $d$-compactness is given by

$$
\left\{S_{n}\right\} d \text {-compact and }\left\{S_{n}\right\}^{*} \subset S \Rightarrow S_{n} \rightarrow S \text {. }
$$

For $K, K_{n}: X \rightarrow Y, K_{n} \rightarrow K$ represents pointwise convergence, that is, $K_{n} x \rightarrow K x$ for $x \in X$ as $n \rightarrow \infty$, and $K_{n} \stackrel{c}{\rightarrow} K$ represents continuous convergence, that is, $x_{n} \rightarrow x$ implies $K_{n} x_{n} \rightarrow K x$ for all $x \in X$.

A word of caution: here and in what follows, the symbols " $\rightarrow$ " and “ $\stackrel{w}{\rightarrow}$ " shall denote, respectively, the strong convergence and the weak convergence.

\section{REGULAR OPERATOR APPROXIMATION THEORY}

Let us consider operators $A, A_{n}: X \rightarrow Y$. An operator $A$ is called regular if $\left\{x_{n}\right\}$ bounded and $\left\{A x_{n}\right\} d$-compact implies $\left\{x_{n}\right\} d$-compact. An operator sequence $\left\{A_{n}\right\}$ is asymptotically regular if $\left\{x_{n}: n \in N^{\prime}\right\}$ bounded and $\left\{A_{n} x_{n}: n \in N^{\prime}\right\} \quad d$-compact implies $\left\{x_{n}: n \in N^{\prime}\right\} d$-compact. The regular convergence $\left(A_{n} \stackrel{r}{\rightarrow} A\right)$ is defined as follows: $A_{n} \stackrel{r}{\rightarrow} A$ if $A_{n} \stackrel{c}{\rightarrow} A$ and $\left\{A_{n}\right\}$ is asymptotically regular. We note that $A_{n} \stackrel{c}{\rightarrow} A$ implies that $A$ is continuous, and $A_{n} \stackrel{r}{\rightarrow} A$ implies that $A$ is regular and continuous. By simple arguments we find that $\left\{A_{n}\right\}$ asymptotically regular and $\left|\lambda_{n}\right| \geq \delta>0$ for all $n \geq n_{0}$ implies that $\left\{A_{n} \lambda_{n}\right\}$ is asymptotically regular, and $A_{n} \stackrel{r}{\rightarrow} A$ and $\lambda_{n} \rightarrow \lambda \neq 0$ implies that $\lambda_{n} A_{n} \stackrel{r}{\rightarrow} \lambda A$.

To include some more definitions, let $X$ be a real Banach space with its dual $X^{*}$ uniformly convex. For $n \in N$, let $P_{n}$ be the projection on $X$ with $P_{n} X=X_{n}, \operatorname{dim} X_{n}<\infty$, and $P_{n} \rightarrow I$ as $n \rightarrow \infty$. Then $\left\{P_{n}\right\}$ is uniformly bounded, $P_{n} \stackrel{c}{\rightarrow} I$, that is, $P_{n} x_{n} \rightarrow x$ as $x_{n} \rightarrow x$, and the convergence in $P_{n} \rightarrow I$ is uniform on a compact set. Let $J: X \rightarrow X^{*}$ be a normalized duality mapping, that is, $[x, J x]=\|x\|^{2}$ and $\|J x\|=\|x\|$, where $[\cdot,, \cdot]$ is the duality pairing between the elements of $X$ and $X^{*}$. Here $P_{n}^{*}$ is a projection on $X^{*}$ with $P_{n}^{*} \rightarrow I^{*}$ as $n \rightarrow \infty$.

An operator $A: X \rightarrow X$ is said to be strongly accretive if, for a constant $\alpha>0$,

$$
[A x-A y, J(x-y)] \geq \alpha\|x-y\|^{2} \quad \text { for all } x, y \in X .
$$

We note that $(2.1)$ also implies that

$$
|[A x-A y, J(x-y)]| \geq \alpha\|x-y\|^{2} \quad \text { for all } x, y \in X .
$$

Next, we shall need to recall a theorem of Anselone and Ansorge [2] crucial to the work at hand. The scope of the theorem extends to the case of compact operators as well.

Lemma 2.1 [2, Theorem 4.10]. Let $X$ and $Y$ be two Banach spaces and $A$, $A_{n}: X \rightarrow Y$ be operators from $X$ to $Y$. If $A_{n} \stackrel{r}{\rightarrow} A, y_{n} \rightarrow y, \gamma>0$, and

$$
\begin{aligned}
S & =\{x \in X: A x=y,\|x\| \leq \gamma\}, \text { and } \\
S_{n} & =\left\{x_{n} \in X: A_{n} x_{n}=y_{n},\left\|x_{n}\right\| \leq \gamma\right\},
\end{aligned}
$$

then $\left\{S_{n}\right\}$ is d-compact, $\left\{S_{n}\right\}^{*} \subset S$, and $S_{n} \rightarrow S$. 
Lemma 2.2 [11, Lemma 18.2]. Let $R^{n}$ be a real Euclidean space with the inner product $\langle\cdot, \cdot\rangle$. Assume $D \subset R^{n}$ is bounded, open, and convex, and $0 \in D$. Assume $F: \bar{D} \rightarrow R^{n}$ is continuous and $\langle F x, x\rangle>0$ for $x \in \delta D$. Then $F x=0$ for some $x \in D$.

Lemma 2.3 [16, Proposition]. Let $X$ be a separable Banach space with $X^{*}$ uniformly convex, and let $\left\{x_{n}\right\}$ be a bounded sequence in $X$. Then there exist a subsequence $\left\{x_{k}\right\}$ (say) and a point $v \in X$ such that $\left\{J\left(x_{n}-v\right)\right\} \stackrel{w}{\rightarrow} 0$ in $X^{*}$.

Now we compare the equations

$$
\begin{cases}A x=b, & x, b \in X, \\ P_{n} A x_{n}=P_{n} b, & x_{n} \in X_{n}, b \in X .\end{cases}
$$

Here

$$
\begin{aligned}
P_{n} A x_{n}=P_{n} b & \Leftrightarrow\left[P_{n}\left(A x_{n}-b\right), P_{n}^{*} J x\right] \\
& =\left[P_{n}\left(A x_{n}-b\right), J x\right] \\
& =\left[A x_{n}-b, P_{n}^{*} J x\right] \\
& =\left[A x_{n}-b, J x\right]=0 \quad \text { for } x \in X_{n} .
\end{aligned}
$$

We are about to consider the main results.

Theorem 2.4. Let $A: X \rightarrow X$ be bounded, continuous, and strongly accretive with a constant $\alpha>0$. If $P_{n}$ is projection on $X$ with $X_{n}=P_{n} X$ and $J: X \rightarrow$ $X^{*}$ is a normalized duality mapping, where $X^{*}$ is uniformly convex, then the following implications hold:

(i) For $b \in X,\|J x\|=\|x\| \geq \gamma>\|A(0)-b\| / \alpha, x \in X, \gamma>0$,

$$
[A x-b, J x]>0 \text {. }
$$

(ii) For $b \in X, \gamma>0$, and $[A x-b, J x]>0$ with $\|x\|=\gamma$, we have

$$
P_{n} A x_{n}=P_{n} b \quad \text { for some } x_{n} \in X_{n} \text { with }\left\|x_{n}\right\|<\gamma, n \in N \text {. }
$$

(iii) $P_{n} A \stackrel{r}{\rightarrow} A$.

(iv) For $b \in X$ and $\gamma>\|A(0)-b\| / \alpha$,

$$
S_{n}=\left\{x_{n} \in X_{n}: P_{n} A x_{n}=P_{n} b,\left\|x_{n}\right\| \leq \gamma, n \in N\right\} \neq \varnothing .
$$

(v) The equation $A x=b$ has a unique solution $x$ with $\|x\| \leq \gamma$.

(vi) $S_{n} \rightarrow\{x\}$.

Proof. (i)

$$
\begin{aligned}
{[A x-b, J x] } & =[A x-A(0), J x]+[A(0)-b, J x] \\
& \geq \alpha\|x\|^{2}-\|A(0)-b\|\|x\| \\
& >\alpha\|x\|^{2}-\alpha \gamma\|x\| \geq 0 .
\end{aligned}
$$

(ii) With no loss of generality, we take $\operatorname{dim} X_{n}=n$. Let $\left\{\phi_{1}, \ldots, \phi_{n}\right\}$ be any basis of $X_{n}$. Then

$$
J x_{n}=\sum_{i=1}^{n} a_{i}^{n} J \phi_{i}, \quad x_{n} \in X_{n} .
$$


The correspondence $x_{n} \leftrightarrow\left(a_{1}^{n}, \ldots, a_{n}^{n}\right)$ defines an isomorphism $X_{n} \leftrightarrow R^{n}$. Let us define $F: R^{n} \rightarrow R^{n}$ by

$$
F a^{n}=\left(\left[A x_{n}-b, J \phi_{1}\right], \ldots,\left[A x_{n}-b, J \phi_{n}\right]\right) .
$$

Then $F$ is continuous, and by (2.3) we have

$$
F a^{n}=0 \Leftrightarrow P_{n} A x_{n}=P_{n} b .
$$

Next, let $D_{n}=\left\{x_{n} \in X_{n}:\left\|x_{n}\right\|<\gamma\right\}$ and $D_{n} \leftrightarrow D^{n} \subset R^{n}$. Then $D^{n}$ is bounded, open, and convex, and $0 \in D^{n}$. Also, $\delta D_{n} \leftrightarrow \delta D^{n}$. By hypothesis,

$$
\left[F a^{n}, a^{n}\right]=\left[A x_{n}-b, J x_{n}\right]>0 \text { for } a^{n} \in \partial D^{n} \text {. }
$$

We obtain, by Lemma 2.2, $F a^{n}=0$ for $a^{n} \in D^{n}$. Hence there exists $J x_{n} \leftrightarrow a^{n}$ such that $x_{n} \in D_{n}$ and $P_{n} A x_{n}=P_{n} b$.

(iii) Since $P_{n} \stackrel{c}{\rightarrow} I$ implies $P_{n} A \stackrel{c}{\rightarrow} A$, it suffices to show that $\left\{P_{n} A\right\}$ is asymptotically regular. To achieve this, let $\left\{x_{n}\right\}$ be bounded and $\left\{P_{n} A x_{n}\right\}$ $d$-compact. Then $\left\{A x_{n}\right\}$ is bounded. Since $P_{n} A x_{n} \rightarrow b$ for some $b \in X$, $n \in N^{\prime \prime} \subset N^{\prime}$ and, by Lemma 2.3, $J\left(x_{n}-x\right) \stackrel{w}{\rightarrow} 0$ for some $x \in X, n \in N^{\prime} \subset N$, it follows that

$$
\begin{gathered}
{\left[A x, J\left(x_{n}-x\right)\right] \rightarrow 0,} \\
{\left[A x_{n}, P_{n}^{*} J\left(x_{n}-x\right)\right]=\left[P_{n} A x_{n}, J\left(x_{n}-x\right)\right] \rightarrow 0,}
\end{gathered}
$$

and

$$
\left[A x_{n}-A x, J\left(x_{n}-x\right)\right]=\left[A x_{n}, J\left(x_{n}-x\right)\right]-\left[A x, J\left(x_{n}-x\right)\right] \rightarrow 0 .
$$

Since $A$ is strongly accretive, by condition (2.1), we get

$$
\alpha\left\|x_{n}-x\right\|^{2} \leq\left[A x_{n}-A x, J\left(x_{n}-x\right)\right] \rightarrow 0 .
$$

Therefore, $x_{n} \rightarrow x$ for $n \in N^{\prime \prime}$, and, consequently, $\left\{x_{n}\right\}$ is $d$-compact and $\left\{P_{n} A\right\}$ asymptotically regular.

(iv) Assertions (i) and (ii) imply that $S_{n} \neq \varnothing$.

(v) Since $A$ is strongly accretive, this implies that any solution $x$ of $A x=b$ is unique.

(vi) Since $P_{n} A \stackrel{r}{\rightarrow} A$ (assertion (iii)) and $P_{n} b \rightarrow b$ (since $P_{n} \rightarrow I$ ), we obtain from Lemma 2.1 and assertion (v) that $S_{n} \rightarrow\{x\}$.

\section{ApPlication to NUMERICAl RANGES}

This section deals with regular approximation of nonlinear equations involving the numerical ranges of Banach space operators.

We define the numerical range of an operator $A: X \rightarrow X$, denoted $n[A]$, by

$$
n[A]=\left\{\frac{[A x-A y, J(x-y)]}{\|x-y\|^{2}}: x, y \in X, x \neq y\right\} .
$$

The numerical range $n[A]$ has properties similar to those of the Zarantonello numerical range $N[A]$, defined as [17]

$$
N[A]=\left\{\frac{\langle A x-A y, x-y\rangle}{\|x-y\|^{2}}: x, y \in X, x \neq y\right\},
$$

where $X$ is a Hilbert space and $\langle\cdot, \cdot\rangle$ is the standard inner product on $X$. Clearly, $n[A]$ reduces to $N[A]$ when $X$ is a Hilbert space. Let us describe some of the elementary properties of $n[A]$. 
Theorem 3.1. Let $A, B: X \rightarrow X$ be mappings from a real Banach space $X$ into itself and $\lambda \in K$ (field). Then

(i) $n[\lambda A]=\lambda n[A]$,

(ii) $n[A+B] \subseteq n[A]+n[B]$, and

(iii) $n[A-\lambda I]=n[A]-\{\lambda\}$.

Proof. The proof follows from the definition.

Theorem 3.2. Let $X$ be a real separable Banach space and its dual $X^{*}$ uniformly convex. If $A: X \rightarrow X$ is bounded and continuous, and a number $\lambda \in K$ is at a positive distance $\alpha$ from the numerical range $n[A]$ of $A$, that is,

$$
\alpha=\inf \{|\lambda-\mu|: \mu \in n[A]\}>0,
$$

then the following implications hold.

(i) If $b \in X,\|x\| \geq \gamma>\|(A-\lambda I)(0)-b\| / \alpha$ for some $x \in X, \gamma>0$, then $[(A-\lambda I) x-b, J x]>0$.

(ii) For $b \in X, \gamma>0$, and $[(A-\lambda I) x-b, J x]>0$ with $\|x\|=\gamma$, we have $P_{n}(A-\lambda I) x_{n}=P_{n} b$ for some $x_{n} \in X_{n},\left\|x_{n}\right\|<\gamma, n \in N$.

(iii) $P_{n}(A-\lambda I) \stackrel{r}{\rightarrow}(A-\lambda I)$.

(iv) For $b \in X$ and $\gamma>\|(A-\lambda I)(0)-b\| / \alpha$, we have

$$
S_{n}=\left\{x_{n} \in X_{n}: P_{n}(A-\lambda I) x_{n}=P_{n} b,\left\|x_{n}\right\| \leq \gamma, n \in N\right\} \neq \varnothing .
$$

(v) The equation $(A-\lambda I) x=b$ has a unique solution $x$ for $\|x\| \leq \gamma$.

(vi) $S_{n} \rightarrow\{x\}$.

Proof. Since the proof follows from an application of Theorem 2.4, it would suffice to show that $A-\lambda I$ is strongly accretive. For $x, y \in X, x \neq y$, we get

$$
\begin{aligned}
\mid[(A & -\lambda I) x-(A-\lambda I) y, J(x-y)] \mid \\
& =|[A x-A y, J(x-y)]-\lambda[x-y, J(x-y)]| \\
& =\mid\left[\frac{[A x-A y, J(x-y)]}{\|x-y\|^{2}}-\lambda \mid\|x-y\|^{2}\right. \\
& \geq \alpha\|x-y\|^{2} .
\end{aligned}
$$

Remark 3.3. Theorem 3.2 reduces to the case of the Zarantonello numerical range when $X$ is a Hilbert space.

\section{ACKNOWLEDGMENT}

The author wishes to express his sincere appreciation to the referee and Professor Dale E. Alspach for some suggestions leading to the revised version.

\section{REFERENCES}

1. P. M. Anselone, Collectively compact operator approximation theory, Prentice-Hall, Englewood Cliffs, NJ, 1971.

2. P. M. Anselone and R. Ansorge, Compactness principles in nonlinear operator approximation theory, Numer. Funct. Anal. Optim. 1 (1979), 589-618.

3. $ـ, A$ unified framework for the discretization of nonlinear operator equations, Numer. Funct. Anal. Optim. 4 (1981), 61-99.

4. P. M. Anselone and Jin-Gan Lei, The approximate solution of monotone nonlinear operator equations, Rocky Mountain J. Math. 16 (1986), 791-801. 
5. $ـ$, Nonlinear operator approximation theory based on demi-regular convergence, Acta Math. Sci. (English ed.) 6 (1986), 121-132.

6. R. Ansorge and Jin-Gan Lei, The convergence of discretization methods if applied to weakly formulated problems: Theory and examples, Z. Angew. Math. Mech. 71 (1991), 201-221.

7. K. E. Atkinson, The numerical solution of a bifurcation problem, SIAM J. Numer. Anal. 14 (1977), 584-599.

8. R. D. Grigorieff, Über diskrete approximetionen nichtlinearer Gleichungen. I, Math. Nachr. 69 (1975), 253-272.

9. M. A. Krasnoselskii et al., Approximate solutions of operator equations, Wolters-Noordhoff, Groningen, 1972.

10. M. A. Krasnoselskii, Topological methods in the theory of nonlinear integral equations, MacMillan, New York, 1964.

11. M. M. Vainberg, Variational method and method of monotone operators in the theory of nonlinear equations, Wiley, New York, 1973.

12. R. U. Verma, On the external approximation-solvability of nonlinear equations, Panamer. Math. J. 2 (1992), 23-42.

13. $\_$Phi-stable operators and inner approximation-solvability, Proc. Amer. Math. Soc. 117 (1993), 491-499.

14. $\ldots$, General approximation-solvability of nonlinear equations involving A-regular operators, Appl. Math. Lett. 6 (1993), 31-33.

15. _ـ On regular operator approximation theory, J. Math. Anal. Appl. 183 (1994), 591-604.

16. J. R. Webb, On a property of duality mappings and the A-properness of accretive operators, Bull. London Math. Soc. 13 (1981), 235-238.

17. E. Zarantonello, The closure of the numerical range contains the spectrum, Bull. Amer. Math. Soc. 70 (1964), 781-787.

18. E. Zeidler, Nonlinear functional analysis and its applications II/B, Springer-Verlag, New York, 1990.

Department of Mathematics, University of Central Florida, Orlando, Florida 32816 Current address: International Publications, 12046 Coed Drive, Orlando, Florida 32826 